\title{
Physical therapy management of a rare multi-ligamentous knee injury following dislocation with a focus on gait training and therapeutic strengthening
}

Ethan Griffel, Alex Urfer* and James Ralphs

*Correspondence: urfealex@isu.edu

CrossMark

$\leftarrow$ Click for updates

Idaho State University, Department of Physical and Occupational Therapy, Pocatello, Idaho 83209, USA.

\begin{abstract}
Background: Knee dislocation is a traumatic but relatively rare injury that generally results in damage to multiple major stabilizing ligaments of the knee and, at times, vascular and neural compromise. Likewise, a rupture of the patellar tendon is extremely debilitating and rare. When sustained collectively these injuries can lead to difficulty in not only surgical intervention but also the rehabilitation processes. These injuries cause major impairments that must be addressed in a somewhat sequential manner to limit the development of long term difficulties and improve the prognosis for patients. The purpose of this case is to demonstrate the post-surgical physical therapy management of one individual with this injury.

Case presentation: The patient was a 42-year-old male who presented post-operatively following right knee dislocation and patellar ligament disruption without vascular or neural disruption. Following surgical repair of the medial collateral ligament and patellar ligament, the patient began physical therapy. The patient's deficits included decreased range of motion, mobility, and strength of his right lower extremity. Therapeutic exercise, neuromuscular reeducation, gait training and modalities were provided to address the patient's impairments and functional limitations.

Discussion: This case study follows the patient's first 10 weeks of rehabilitation following surgery in an outpatient setting. The patient demonstrated rapid recovery with minimal to no pain and achieved all goals set for him in that time frame. He improved his functional ability through increased muscular strength and endurance, improved gait mechanics and balance, improved knee flexion range of motion by 50 degrees and increased Lower Extremity Functional Scale score by 28 points.

Conclusion: Overall, a physical therapy approach for this rare injury involving a slow increase in range of motion and gradual introduction of resistance exercise, balance, and gait training, improved the patient's impairments and functional limitations.
\end{abstract}

Keywords: Multi-ligamentous Knee Injury, Patellar Ligament Disruption, Knee Dislocation, Therapeutic Exercise

\section{Background}

Multi-ligamentous knee injuries (MLKI) result when there is damage to two or more of the major ligaments of the knee $[1,2]$. Of the multiple classification systems used in identifying severity of this injury, the most common appears to follow the Schenck knee dislocation classification $[\mathbf{1 , 2}]$. This system ranks knee dislocations based upon the number of ligaments disrupted secondary to the trauma. It ranges from knee dislocation (KD) I to KD V with the most common classification being KD III which involves damage to the anterior cruciate ligament $(\mathrm{ACL})$, posterior cruciate ligament $(\mathrm{PCL})$, and either the medial or lateral posterior corners or one of the collateral ligaments $[1,2]$. 
Griffel et al, Physical Therapy and Rehabilitation 2018,

The incidence at which either a KD/MLKI or patellar ligamentdisruption (PLD) occurs is exceedingly rare. According to Arom et al., the demographic data associated with KD, indicate the incidence to be .072 cases per every 100 patients seen for orthopedic injuries in a year with males only slightly more prone than females (.076 per 100 to .069 per 100 respectively) [3]. Likewise, the rates of PLD are also very low. Clayton and colleagues placed the incidence of PLD at .68 per every 100 thousand individuals [4]. Although the literature is limited on specific incidence rates of sustaining these injuries concurrently, we can infer based on the low rates of each injury independently that it is extremely low. Further, these injuries are shown to affect younger adults of mean age $35[1,3,5]$ most often and are a result of motor vehicle accidents, athletic injuries, or falls from lower than 5 feet [1].

The presentation of this injury can be difficult to diagnosis, and the clinician must be aware of associated damage to other tissues. While KD will frequently spontaneously reduce prior to medical evaluation $[2,5]$, the injury will have significant joint effusion and adjacent edema [6], as well as severe joint laxity in most planes of motion [6]. Significant pain and weakness of the knee is also present [7]. Finally, deformity at the knee will be noticeable with and without reduction of the joint. If reduction has occurred and PLD is present, a palpable gap will be noticed at the inferior pole of the patella while the patella will be located slightly superiorly $[\mathbf{6}, \mathbf{7}]$. When examining a patient with suspected KD it is important to check the integrity of the neurovascular structures supplying the lower limb below the knee as it has been shown that there is a high incidence of neurovascular compromise associated with KD especially when occurring in the posterior direction [1]. Possible tests to check for neurovascular compromise could include pedal pulses, ankle brachial index, sensation testing, and observation of color.

Surgical management of such injuries is varied both in timeline to perform surgery as well as the operative procedure. The literature suggests examples of both acute and chronic timelines to surgery defined as within 3 weeks of injury or after 3 weeks from injury [2]. Mook suggests the latter timeline results in less range of motion (ROM) deficits when compared to the former [8]. Further, surgery is undertaken in either a single or double stage repair [2]. Two stage repairs are more common today and made necessary due to differing postsurgical rehabilitation protocols associated with the different structures needing repair depending on the injury pattern $[2,9,10]$. With a KD and concurrent PLD the first surgery generally addresses the patellar ligament as well as any damaged collateral ligaments and a second surgery will be performed after return of full ROM and quad strength to address any other damaged structures $[\mathbf{9}, \mathbf{1 1}]$.

Current physical therapy management guidelines for such an injury are lacking in specifics of best practice. While protocols for rehabilitation for cruciate ligament repairs are common there is little evidence to suggest a process for rehabbing a
$\mathrm{KD} / \mathrm{PLD}$ injury besides progressing ROM and strength of the affected limb while protecting the surgical site $[6,12]$. Further, the literature is unclear on the timeline to mobilization following PLD surgery with some reports advocating prolonged immobilization while others support early controlled passive range of motion (PROM) $[13,14]$.

Knee dislocations and multi-ligamentous knee injuries are exceedingly rare and underrepresented in the literature concerning lower extremity (LE) orthopedic conditions. Add PLD to this already traumatic injury and examples in the literature are almost non-existent. The purpose, therefore, of this case report is to add to the paucity of literature concerning this injury pattern and give a successful example of how one such injury was rehabilitated during a 10-week period during the acute phases following surgery.

\section{Case presentation \\ Subjective examination}

This patient was a 42-year-old male who sustained a downhill skiing accident in the early season resulting in a dislocation of his right $(R)$ knee. The injury spontaneously reduced and upon medical evaluation by a physician, it was determined he had total ruptures of the patellar ligament (PL), medial collateral ligament $(\mathrm{MCL})$, and the posterior cruciate ligament $(\mathrm{PCL})$. In addition, there was increased laxity of the anterior cruciate ligament (ACL). This patient's surgeon opted for a delayed two stage surgical intervention with the initial surgery taking place 3 weeks after initial the injury addressing the repair of the MCL and PL. The patient presented to the outpatient clinic with bilateral axillary crutches and physician orders for nonweight bearing (NWB) activities on the R for six weeks, use of a continuous passive motion (CPM) machine from $0-20^{\circ}$, and use of a knee immobilizing brace locked at $0^{\circ}$ extension anytime he was not in the CPM.

The patient was a professor of geology who was actively engaged in skiing, mountain biking, and hiking prior to his accident. Further, he spent time in the field and behind the desk as part of his work duties. He reported experiencing no pain when he presented to the clinic. The patient's medications included Oxycodone from which he abstained, Ibuprofen as needed, and Aspirin. The patient's reported goals were to return to normal functional and recreational activities and unimpaired mobility. A review of systems for this patient was unremarkable, and the only reported past medical injury included fractured ribs and clavicle following a mountain bike accident greater than 10 years in the past. The patient also reported multiple ankle injuries from soccer that he identifies as the source of his decreased ankle ROM.

\section{Objective examination}

A systems review for this patient consisting of deep tendon reflexes, pathological reflexes, and dermatomal sensation was unremarkable. A screen of the patient's lower quarter function indicated severely limited ROM and strength of the RLE. 
Griffel et al, Physical Therapy and Rehabilitation 2018,

Table 1 Provides the results of the lower quarter musculoskeletal screen.

Table 1. Systems Review/Screen.

\begin{tabular}{lllll}
\hline LE & \multicolumn{2}{l}{ Active ROM (AROM) } & \multicolumn{2}{l}{ Resistance Testing } \\
\cline { 2 - 5 } & Right & Left & Right & Left \\
\hline Hip & WNL, Flexion deferred & WNL & Deferred & S/PF \\
Knee & Limited Flexion & WNL & Deferred & S/PF \\
Ankle & Limited DF & Limited DF & S/PF & S/PF \\
\hline
\end{tabular}

$\mathrm{S} / \mathrm{PF}=$ Strong and Pain Free, $\mathrm{WNL}=$ Within normal limits

Palpation examination of the patient revealed increased edema of the $\mathrm{R}$ knee. A girth measurement of 45 centimeters $(\mathrm{cm})$ was taken at the joint line. Additionally, the patient demonstrated slight tenderness to palpation at the $\mathrm{R}$ distal vastus medialis obliquus (VMO) as well as decreased cutaneous sensation to light touch of the distal anterior femoral cutaneous nerve distribution. Joint play assessment of the tibiofemoral joint was deferred at initial evaluation. However, decreased joint ROM was noted.

Objective strength testing of the patient was deferred due to acuteness of surgery and physician's orders. Active range of motion (AROM) measurements revealed severely compromised ROM of the R knee and normal L knee ROM. Initial ROM measurements taken with a universal goniometer reported the following knee motion: $\mathrm{R}=0^{\circ}-15^{\circ}$ and $\mathrm{L}=0^{\circ}-144^{\circ}$. PROM measurements were not taken do to the acuteness of the injury/surgical repair and patient apprehension.

A Lower Extremity Functional Scale (LEFS) [15] was administered to assess the patient's subjective feelings of limitation regarding his R LE. The LEFS has been shown to have excellent test-retest reliability as well as good validity and identifies nine points as the minimal clinically important difference [15]. The LEFS score at initial examination was a 27/80 indicating a high level of subjective limitation of the R LE. Additional tests and measures were planned to assess the patient's balance, but were postponed until the patient returned to full weight-bearing status (FWB).

\section{Evaluation and Physical Therapy Diagnosis}

The results of the examination indicated a physical therapy diagnosis of impaired functional ability and mobility due to decreased strength and ROM of R LE, impaired gait mechanics, and fatigue secondary to surgical repair of multiple ligaments of $\mathrm{R}$ knee following a traumatic knee dislocation. The prognosis was favorable for the patient to return to functional mobility and activity given the shown benefits of common physical therapy interventions for treating MLKI and other knee injuries such as ACL repairs [16-18]. The following goals were set for therapy:

1. Patient will demonstrate improved R knee AROM to 0-90 degrees in three weeks

2. Patient will demonstrate ability to ascend and descend one flight of stairs without antalgic gait or mechanical compensation and one handrail assistance in eight weeks.

3. Patient will report walking one mile on flat stable surface without pain, fatigue, or assistive device (AD) in eight weeks.

4. Patient will report improved LEFS score to 50/80 in eight weeks

\section{Interventions}

The timeline of this case report covers 10 weeks of post-surgical rehabilitation for the patient following an initial three-week period which focused solely on controlling edema and implementing a home exercise plan (HEP) to maintain strength of muscle groups adjacent to the R knee. See Table 2 for full HEP covering these 10 weeks. A plan of care was developed to see the patient in clinic once a week until WB and twice a week once FWB was attained. The patient was seen for 17 treatment sessions over this report.

Table 2. Home Exercise Plan (HEP).

\begin{tabular}{|c|c|c|c|c|}
\hline Exercise & Sets & Reps & Frequency & Initiation \\
\hline Quad sets & 2 & 12 & $2 \mathrm{x}$ day & $\begin{array}{l}\text { Beginning } \\
\text { week } 1\end{array}$ \\
\hline Towel calf stretch & 3 & $\begin{array}{l}20 \mathrm{sec} \\
\text { holds }\end{array}$ & $2 \mathrm{x}$ day & $\begin{array}{l}\text { Beginning } \\
\text { week } 1\end{array}$ \\
\hline Resisted DF/PF (RTB) & 2 & 12 & $2 x$ day & $\begin{array}{l}\text { Beginning } \\
\text { week } 1\end{array}$ \\
\hline Hip $\mathrm{ABd}$ /ext with brace on & 2 & 12 & $2 x$ day & $\begin{array}{l}\text { Beginning } \\
\text { week } 1\end{array}$ \\
\hline Towel slides/Seat flex & 2 & 15 & $3 x$ day & $\begin{array}{l}\text { Beginning } \\
\text { week } 6\end{array}$ \\
\hline Partial squat (60 degrees) & 2 & 10 & $2 x$ day & $\begin{array}{l}\text { Beginning } \\
\text { week } 6\end{array}$ \\
\hline Bike no resistance & 1 & $5-10 \mathrm{~min}$ & $1 \mathrm{x}$ day & $\begin{array}{l}\text { Beginning } \\
\text { week } 7\end{array}$ \\
\hline SLR weight at knee & 2 & 12 & $2 x$ day & $\begin{array}{l}\text { Beginning } \\
\text { week } 7\end{array}$ \\
\hline SLR weight at ankle & 2 & 10 & $2 x$ day & $\begin{array}{l}\text { Beginning } \\
\text { week } 10\end{array}$ \\
\hline Leg press & 3 & 10 & $\begin{array}{l}\text { 1x week other } \\
\text { than in clinic }\end{array}$ & $\begin{array}{l}\text { Beginning } \\
\text { week } 10\end{array}$ \\
\hline
\end{tabular}

During these first weeks, ROM was progressed solely using the CPM which the patient had previously been using. Grade II patellar mobilizations were also used in the medial and lateral directions to begin improving mobility of the patella. Initial strengthening exercises performed, focused on adjacent joint musculature to the $\mathrm{R}$ knee until appropriate healing had occurred at the PL repair. These activities included progressive resistance exercises (PRE) such as 4-way ankle and hip abduction and extension in standing. Straight leg raises were introduced with resistance at the knee and progressed both in resistance and altering the resistance lever arm to the ankle. Various hip abductor and external rotation exercises included side-lying clamshells and leg-raises. As the patient progressed to WB, more functional activities were performed 
Griffel et al, Physical Therapy and Rehabilitation 2018,

such as squats, double- and single-leg leg press, calf-raises, crab-walks with a resistance band around the knees, and single-leg step ups onto an 8-inch platform and eccentric lowering. Once the patient demonstrated knee flexion of $105^{\circ}$, stationary biking was introduced with no resistance for approximately five minutes at the start of therapy sessions with the intent of increasing resistance when the patient achieved 20 minutes of continuous cycling.

With initiation of weight bearing activity and ambulation, the patient immediately demonstrated an abnormal gait pattern consisting of decreased tibial advancement in terminal swing on the $\mathrm{R}$, decreased heel lift in pre-swing on the $\mathrm{R}$, decreased knee flexion with pre-swing on the $\mathrm{R}$, and shortened step length on the R. Gait training was progressed in a typical fashion beginning with $50 \%$ WB on the R at week 6 and then WB as tolerated at week 7 . It was then progressed by walking with two crutches, walking with a single crutch on the $L$, and finally walking with no AD. We did not have access to parallel bars for gait training. Verbal cues were used to decrease gait deviations noted previously as well as visual cuing by having the patient walk towards a mirror and examine his gait.

During gait training the patient demonstrated most balance impairments when in a decreased base of support such as single leg stance phases of gait. Sharpened Romberg [18] testing and single leg stance test (SLS) [19] were performed at treatment week seven with results shown in Table 3. As the patient had limitations in balance testing, balance training was initiated progressing from tandem on a firm surface and eyes open (EO) to eyes closed (EC). Single leg stance also began on a firm surface with $E O$ and progressed to EC then to a compliant surface with EO and finally with EC. Once the patient had progressed far enough in these activities new ones were introduced such as single leg stance with points marked in a circle around the patient that he was instructed to reach out and tap with the toe of his left foot. These activities were designed to stress the patient's static/dynamic control, limits of stability, and muscular control of the R LE. Once performed well enough on a firm surface the patient also performed this activity on a therapeutic mini trampoline. Balance activities were always performed near a support bar to insure patient safety.

Table 3. Initial Balance Testing.

\begin{tabular}{lll}
\hline Outcome Measure & $\mathbf{R}$ foot forward EC & $\mathbf{L}$ foot forward EC \\
\hline Sharpened Romberg & 15 seconds & 8 seconds \\
\hline Outcome measure & Right & Left \\
\hline Single Leg Stance & 6 seconds & 20 seconds \\
\hline
\end{tabular}

\section{Outcomes}

The patient presented in this report completed 10 weeks of therapy for a total of 17 visits. The patient achieved all goals previously defined in the set timeframe and by treatment week 10 the patient had returned to FWB status without an AD. During ambulation, the patient demonstrated no abnormal gait patterns and ambulated greater than one mile. The patient demonstrated observable improvements in LE strength in the absence of objective measurements by performing functional activities such as FWB squats and step ups. Additionally, the patient increased the amount of resistance managed with LE exercises, further indicating an increase in strength.

The patient showed improvement in ROM of the R knee of $104^{\circ}$ in flexion. There was a decrease in circumferential measurements at the knee joint line of $1.5 \mathrm{~cm}$, and an improvement in the balance assessments of Sharpened Romberg and SLS. The patient had improved subjective ratings via the LEFS of 28 points easily surpassing the minimal clinically important difference of nine points. Table 4 shows the pre- and postmeasurements for all outcome measures taken for this patient over 10 weeks.

Table 4. Pre- and Post-Measurements.

\begin{tabular}{lll}
\hline Measure & Treatment week $\mathbf{1}$ & $\begin{array}{l}\text { Treatment week } \\
\mathbf{1 0}\end{array}$ \\
\hline R knee AROM (degrees) & $0-15$ & $0-119$ \\
LEFS & $27 / 80$ & $55 / 80$ \\
\hline Sharpened Romberg (sec) & NT(not tested) & $\begin{array}{l}\text { R foot forward }=20 \\
\text { L foot forward }=14\end{array}$ \\
\hline Single leg stance (sec) & NT(not tested) & $\begin{array}{l}\mathrm{R}=13 \\
\mathrm{~L}=20\end{array}$ \\
& & 43.5 \\
\hline $\begin{array}{l}\text { R Knee joint line circumference } \\
(\mathbf{c m})\end{array}$ & 45 & \\
\hline
\end{tabular}

\section{Discussion}

This case report describes the examination and physical therapy treatment of a rare traumatic injury for which the current literature provided little evidence. We were able to effectively manage this patient's condition by following and adapting treatment intervention protocols for similar knee injuries. Our goal with this patient was to foremost avoid a second surgical procedure to repair the PCL and lax ACL by improving muscular strength, ROM, and stability of the joint. To this end, we targeted the newly repaired PL and quadriceps muscle group as well as proximal hip musculature with a strengthening protocol and monitored progression of ROM beginning immediately following surgery.

From what little literature there is pertaining to KD/MLKI, it is clear that early mobilization following the injury is necessary to avoid later difficulties leading to poor functional outcomes. Mook et al. published a systematic review examining retrospective studies covering 396 cases of KD/MLKI [8]. In this review, it was determined that immobilization led to posterior instability in $28 \%$ of patients compared to $12 \%$ of patients mobilized early on in therapy [8]. Additionally, there was reported varus and valgus laxity in $21 \%$ and $26 \%$ of patients respectively compared to $1.6 \%$ and $2 \%$ respectively in patients mobilized early [8]. The guidelines for PLD, however, are much scarcer and contrasting when available. Current 
protocols call for six weeks NWB with complete immobilization [13]. Langenhan and colleagues examine two cohorts of patients following PLD and their study provides evidence suggesting outcomes with early functional mobilization and complete immobilization are similar [20]. One group was allowed no more than $0-40^{\circ}$ of knee flexion and $50 \%$ WB in six weeks while the second group progressed knee flexion from $0^{\circ}$ to $30^{\circ}, 60^{\circ}, 90^{\circ}$ in two week increments and allowed FWB as tolerated from the beginning of therapy. Outcomes between the two groups were not significantly different supporting the case that early mobilization is not detrimental to these patients [20]. Our management of a patient with this injury pattern with early mobilization adds to and supports the literature that early mobilization is beneficial in returning to functional activity in a timely fashion.

Therapeutic exercise $[6,12,16]$ and balance training [17] has been shown to be beneficial for patients recovering from $A C L$ repair and with modification we applied similar protocols to the patient presented to promote strength and stability in the absence of a PCL and full integrity of the ACL. Eitzen and colleagues reported support for a 5-week PRE-program in the acute phase of $A C L$ repair using exercise theories such as open and closed chain and eccentric, concentric, and isokinetic contractions [16]. Adapted principles such as these were paramount in helping us obtain our goal of sufficiently strengthening the knee of this patient to avoid a second surgical procedure. Likewise, Akbari et al. demonstrated the benefits of early balance training following $A C L$ repair [17]. We were able to further demonstrate the benefits of similar balance training in this patient with a more severe injury than an isolated $A C L$ rupture.

\section{Limitations}

The case presented here does have some limitations, foremost among them being that this is the treatment of a single, highly functioning, and active individual. The results obtained may not be achievable within the same timeframe based on associated factors such as activity level and comorbidities of other patients. Additionally, this report covers a limited view of the total plan of care for this patient ending at week ten post-op after which further therapy was performed in a different setting. Also, only functional strength measures were reported as no objective and consistent strength measurement were performed due to the acuteness of injury and tissue healing over the course of treatment.

\section{Conclusion}

As the literature pertaining to injuries like the one presented in this case is limited, it is necessary to use the evidence supporting treating patterns for similar injuries, such as $A C L$, repairs to design an appropriate plan of care for KD/MLKI with concomitant PLD. This case however contributes, to the paucity of literature on the subject, a successful example of how mobilization, therapeutic exercise, and functional activity can be used in the early stages of rehabilitation following a $\mathrm{KD}$ with multi-ligament damage and PLD to promote strength and stability. This is supportive of less invasive and more conservative treatment when there remains damage to $\mathrm{ACL}$ and PCL following an initial surgery.

\section{Competing interests}

The authors declare that they have no competing interests.

Authors' contributions

\begin{tabular}{|l|c|c|c|}
\hline Authors' contributions & EG & AU & JR \\
\hline Research concept and design & -- & -- & -- \\
\hline Collection and/or assembly of data & $\checkmark$ & -- & -- \\
\hline Data analysis and interpretation & $\checkmark$ & $\checkmark$ & $\checkmark$ \\
\hline Writing the article & $\checkmark$ & $\checkmark$ & $\checkmark$ \\
\hline Critical revision of the article & $\checkmark$ & $\checkmark$ & $\checkmark$ \\
\hline Final approval of article & $\checkmark$ & $\checkmark$ & $\checkmark$ \\
\hline Statistical analysis & -- & -- & -- \\
\hline
\end{tabular}

\section{Acknowledgements}

Annie Boal, DPT, OCS and Jocelyn Head, DPT for their guidance during this case.

\section{Publication history}

Editor: Mohammad H. Hadadzadeh, Wheeling Jesuit University, USA. Received: 01-Jan-2018 Final Revised: 18-Mar-2018

Accepted: 28-Mar-2018 Published: 14-Apr-2018

\section{References}

1. Howells NR, Brunton LR, Robinson J, Porteus AJ, Eldridge JD and Murray JR. Acute knee dislocation: an evidence based approach to the management of the multiligament injured knee. Injury. 2011; 42:1198204. | Article | PubMed

2. Cook S, Ridley TJ, McCarthy MA, Gao Y, Wolf BR, Amendola A and Bollier MJ. Surgical treatment of multiligament knee injuries. Knee Surg Sports Traumatol Arthrosc. 2015; 23:2983-91. | Article I PubMed

3. Arom GA, Yeranosian MG, Petrigliano FA, Terrell RD and McAllister $D R$. The changing demographics of knee dislocation: a retrospective database review. Clin Orthop Relat Res. 2014; 472:2609-14. | Article | PubMed Abstract | PubMed FullText

4. Clayton RA and Court-Brown CM. The epidemiology of musculoskeletal tendinous and ligamentous injuries. Injury. 2008; 39:1338-44. | Article I PubMed

5. Gimber LH, Scalcione LR, Rowan A, Hardy JC, Melville DM and Taljanovic MS. Multiligamentous injuries and knee dislocations. Skeletal Radiol. 2015; 44:1559-72. | Article | PubMed

6. Skendzel JG, Sekiya JK and Wojtys EM. Diagnosis and management of the multiligament-injured knee. J Orthop Sports Phys Ther. 2012; 42:234-42. | Article | PubMed

7. Kovacev N, Antic J, Gvozdenovic N, Obradovic M, Vranjes M and Milankov M. Patellar tendon rupture--treatment results. Med Pregl. 2015; 68:228. I PubMed

8. Mook WR, Miller MD, Diduch DR, Hertel J, Boachie-Adjei Y and Hart JM. Multiple-ligament knee injuries: a systematic review of the timing of operative intervention and postoperative rehabilitation. J Bone Joint Surg Am. 2009; 91:2946-57. I Article I PubMed

9. Malvasi S, Gloyeske B, Johnson M and Miller T. Multi-LigamentousKnee Injury in Sports Involving Concomitant Anterior Cruciate Ligament and Patellar Tendon Disruption: A Review of Case Reports. Int J Ath/ Ther Trai. 2016; 21:24-27. | Article

10. Chow FY, Wun YC and Chow YY. Simultaneous rupture of the patellar tendon and the anterior cruciate ligament: a case report and literature review. Knee Surg Sports Traumatol Arthrosc. 2006; 14:1017-20. I Article 
Griffel et al, Physical Therapy and Rehabilitation 2018,

http://www.hoajonline.com/journals/pdf/2055-2386-5-3.pdf

I PubMed

11. Capogna B, Strauss E, Konda S, Dayan A and Alaia M. Distal patellar tendon avulsion in association with high-energy knee trauma: A case series and review of the literature. Knee. 2017; 24:468-476. | Article | PubMed

12. Fanelli GC, Sousa PL and Edson CJ. Long-term followup of surgically treated knee dislocations: stability restored, but arthritis is common Clin Orthop Relat Res. 2014; 472:2712-7. | Article | PubMed Abstract | PubMed FullText

13. Ciriello V, Gudipati S, Tosounidis T, Soucacos PN and Giannoudis PV. Clinical outcomes after repair of quadriceps tendon rupture: a systematic review. Injury. 2012; 43:1931-8. | Article | PubMed

14. Bushnell BD, Tennant JN, Rubright JH and Creighton RA. Repair of patellar tendon rupture using suture anchors. J Knee Surg. 2008; 21:1229. | PubMed

15. Mehta SP, Fulton A, Quach C, Thistle M, Toledo C and Evans NA. Measurement Properties of the Lower Extremity Functional Scale: A Systematic Review. J Orthop Sports Phys Ther. 2016; 46:200-16. | Article I PubMed

16. Eitzen I, Moksnes H, Snyder-Mackler L and Risberg MA. A progressive 5-week exercise therapy program leads to significant improvement in knee function early after anterior cruciate ligament injury. J Orthop Sports Phys Ther. 2010; 40:705-21. | Article | PubMed Abstract | PubMed FullText

17. Akbari A, Ghiasi F, Mir M and Hosseinifar M. The Effects of Balance Training on Static and Dynamic Postural Stability Indices After Acute ACL Reconstruction. Glob J Health Sci. 2015; 8:68-81. | Article | PubMed Abstract | PubMed FullText

18. Raad J. Rehab Measures: Romberg Test. Rehabilitation Measures Database. 2013. | Website

19. Raad J. Rehab Measures: Single leg stance or "One-legged stance test". Rehabilitation Measures Database. 2013. | Website

20. Langenhan R, Baumann M, Ricart P, Hak D, Probst A, Badke A and Trobisch $P$. Postoperative functional rehabilitation after repair of quadriceps tendon ruptures: a comparison of two different protocols. Knee Surg Sports Traumatol Arthrosc. 2012; 20:2275-8. | Article | PubMed

\section{Citation:}

Griffel E, Urfer A and Ralphs J. Physical therapy management of a rare multi-ligamentous knee injury following dislocation with a focus on gait training and therapeutic strengthening. Phys Ther Rehabil. 2018; 5:3. http://dx.doi.org/10.7243/2055-2386-5-3 